\title{
End of the road for adjunctive vitamin $D$ therapy for pulmonary tuberculosis?
}

Or

Adjuctive vitamin D treatment of pulmonary tuberculosis: final nail in the coffin?

\author{
Ibrahim Abubakar*, Frank Kloprogge \\ University College London Institute for Global Health \\ *correspondence: i.abubakar@ucl.ac.uk
}

Adjunctive and host directed therapies are increasingly recognised as an important target in the quest for novel effective antituberculous therapy.(1) The global increase in drug resistance to antibiotics suggests that effective adjunctive medications may provide an alternative avenue to treat infections. Vitamin D supplementation has been hypothesized as a potential adjunctive agent for the treatment of tuberculosis and evaluated in several trials.(2) In this issue of the Journal, Ganmaa and colleagues (2) evaluated the effectiveness of adjunctive vitamin D on sputum culture conversion and assessed the influence of key vitamin D pathway single nucleotide polymorphisms (SNPs) on sputum culture conversion concluding that there is no evidence that vitamin D supplementation improved outcomes.

The most recent Cochrane review on nutritional supplements for patients on tuberculosis treatment(3) found that adjunctive vitamin $D$ therapy does not have any impact on sputum conversion despite multiple studies and the use of various doses. Ganmaa and colleagues provide further evidence, consistent with this previously published review of seven trials which includes 1197 participants,(3)(4) that vitamin D supplementation does not improve sputum culture conversation even among patients with baseline vitamin D deficiency. In keeping with the findings of the study by Ganmaa and colleagues, previous trials also suggest that serum vitamin $D$ level is increased where participants had low baseline vitamin D levels.(3)

Masking of allocation including the use of placebo and recruitment of a sample size with adequate statistical power for the main analysis distinguishes this study from previous attempts to assess the role of vitamin $D$ in sputum culture conversation. The use of directly observed therapy for antituberculosis treatment monitoring gives assurance that patients in the intervention and control arm were adherent. However, assuming that the effect size of vitamin D is modest, even relatively small differences in adherence or availability of antibiotics may alter the observed effect. Therefore monitoring the variability in pharmacokinetics of background therapy which could potentially impact the overall antibacterial effect would have been worthwhile.

A further strength of this study is the subgroup analysis allowing the evaluation of the interaction between important vitamin D pathway SNPs and the effect of supplementation on sputum culture conversion. This is important because previous research(5) suggest that the effect of vitamin $D$ status on outcome is likely to be influenced by variation in SNPs within populations. It is therefore encouraging that the stratified analysis concluded that patients with particular polymorphisms in the gene encoding the vitamin $D$ receptor and the 25 -hydroxyvitamin $D$ 1-alpha hydroxylase enzyme CYP27B1 may benefit from adjuctive vitamin D therapy. The detection of an effect modification by genetic polymorphism may either suggest that in each population there is a smaller subset of individuals who might benefit from vitamin D supplementation or that perhaps the observed effect 
is spurious arising from type 1 error. The difference in the observed polymophisms which affect vitamin D supplementation between this study (2) and previous vitamin D research (5) further negates the hypothesis that specific subsets of the population may benefit from vitamin $D$ therapy. Nevertheless, if further research were to be undertaken on this subject, the relevant subpopulations with a potential to benefit from vitamin $D$ adjunctive therapy would need to be defined in larger cohorts prior to any randomised controlled trial.

While only a secondary outcome, the discernible effect on sputum smear conversation (adjusted HR $1.47,95 \% \mathrm{Cl} 1.09$ to $1.98, \mathrm{P}=0.01$ ) and radiographic resolution might suggest that the effect size on sputum culture conversation and potentially subsequent relapse, if any, is small. Although the study was powered to detect a modest effect, a considerably larger study may be required if the effect of vitamin D supplementation is small; such a small effect may not be clinically valuable given the availability of efficacious antibiotic therapy. Unfortunately too few cases of drug resistant tuberculosis were included to allow an evaluation of the added benefit of vitamin $D$ in patients where the effectiveness of antibiotic therapy is more limited. There is now evidence that short course treatment regimen for fluoroquinolone sensitive multi drug resistant tuberculosis (MDR-TB) achieve high cure rates.(6) The suggestion by Ganmaa and colleages that future evaluation of vitamin $D$ anti-tuberculosis adjunctive therapy should be undertaken in MDR-TB patients may therefore need to focus on fluoroquinolone and/or aminoglycoside resistant patients.

Unfortunately, the inclusion of patients with drug resistant tuberculosis in the trial by Ganmaa and colleagues (2) may also have introduced further bias, although this was adjusted for in the analysis with no consequent change in the observed effect of vitamin $D$ on sputum culture conversion.

Future research on vitamin $D$ adjunctive therapy for tuberculosis should focus on participants with baseline deficiency and genetic polymorphisms associated with evidence of an effect on outcomes to investigate longer term clinical outcomes including relapse within 12 months. (7) It is perhaps time to move on to other adjunctive therapy options.

\section{References}

1. Zumla AI, Gillespie SH, Hoelscher M, Philips PPJ, Cole ST, Abubakar I, et al. New antituberculosis drugs, regimens, and adjunct therapies: needs, advances, and future prospects. Lancet Infect Dis. 2014 Apr;14(4):327-40.

2. Ganmaa D. High-Dose Vitamin D3 During Tuberculosis Treatment in Mongolia: a Randomised Controlled Trial. Am J Respir Crit Care Med. 2017;

3. Grobler L, Nagpal S, Sudarsanam TD, Sinclair D. Nutritional supplements for people being treated for active tuberculosis. Cochrane Database Syst Rev. 2016 Jun 29;(6):CD006086.

4. Nunn A, Phillips PPJ, Abubakar I. Treatment of pulmonary tuberculosis. Curr Opin Pulm Med. 2013 May;19(3):273-9.

5. Martineau AR, Timms PM, Bothamley GH, Hanifa Y, Islam K, Claxton AP, et al. High-dose vitamin $D(3)$ during intensive-phase antimicrobial treatment of pulmonary tuberculosis: a double-blind randomised controlled trial. Lancet. 2011 Jan 15;377(9761):242-50.

6. Aung KJM, Van Deun A, Declercq E, Sarker MR, Das PK, Hossain MA, et al. Successful "9-month Bangladesh regimen" for multidrug-resistant tuberculosis among over 500 consecutive patients. Int J Tuberc Lung Dis Off J Int Union Tuberc Lung Dis. 2014 Oct;18(10):1180-7. 
7. Nunn AJ, Phillips PPJ, Mitchison DA. Timing of relapse in short-course chemotherapy trials for tuberculosis. Int J Tuberc Lung Dis Off J Int Union Tuberc Lung Dis. 2010 Feb;14(2):241-2. 